\title{
NOUVELL
}

\section{Dysfonction mitochondriale et défaillance de la mitophagie dans la maladie d'Alzheimer}

Arnaud Mary ${ }^{\star}$, Loan Vaillant-Beuchot ${ }^{\star}$, Frédéric Checler, Mounia Chami

> La maladie d'Alzheimer est la maladie neurodégénérative la plus répandue dans le monde. En l'absence de thérapie efficace, cette maladie, dont l'incidence ne cesse d'augmenter, représente un défi scientifique, économique, et sociétal. L'un de ses principaux symptômes est la perte de la mémoire, qui se développe sur plusieurs dizaines d'années, associée à une défaillance fonctionnelle de neurones situés dans des zones spécifiques du cerveau régissant les fonctions d'apprentissage et de mémorisation, notamment I'hippocampe. Les analyses post-mortem de cerveaux de patients atteints de la maladie d'Alzheimer, et des modèles murins de la maladie, ont conduit à définir cette maladie comme une «protéinopathie » qui se caractérise par l'accumulation dans les cellules et dans le milieu extracellulaire (s'agrégeant dans des plaques séniles) du peptide amyloïde $\beta(A \beta)$, et par une phosphorylation anormale de la protéine Tau (pTau), conduisant à la dégénérescence neurofibrillaire (neurofibrillary tangles, NFT) des neurones [1]. Le peptide $A \beta$ est produit par clivages successifs de la protéine précurseur du peptide amyloïde (amyloid protein precursor, APP) par les enzymes $\beta$ - et $\gamma$-sécrétases, dans la voie amyloïdogène (Figure 1). L'apparition des formes familiales précoces et agressives de la maladie d'Alzheimer, favorisées par des mutations dans les gènes codant l'APP et les présénilines 1 et 2 (PS1/2, composants du complexe $\gamma$-sécrétase), a conduit à proposer que la voie amyloïdogène jouait un rôle
Université Côte d'Azur, Inserm, CNRS, Institut de pharmacologie moléculaire et cellulaire, Laboratoire d'excellence DistALZ, 660 route des Lucioles, 06560 Sophia-Antipolis, Valbonne, France. ${ }^{*}$ Contribution égale mchami@ipmc.cnrs.fr

majeur dans la pathogenèse de la maladie d'Alzheimer. Néanmoins, l'échec des traitements ciblant uniquement l'A $\beta$ indique que d'autres fragments issus du clivage de I'APP pourraient contribuer aux dysfonctionnements cellulaires impliqués dans le développement de la maladie d'Alzheimer. Le rôle pathogène des fragments $C$-terminaux de l'APP (APP-CTF : C99 et C83) (Figure 1) a ainsi été récemment souligné dans différentes études [2].

Les dysfonctions mitochondriales dans la maladie d'Alzheimer

Les mitochondries sont des organelles intracellulaires dont la fonction principale est de produire de l'énergie sous la forme d'ATP. Dans les neurones, I'ATP est nécessaire notamment à la 


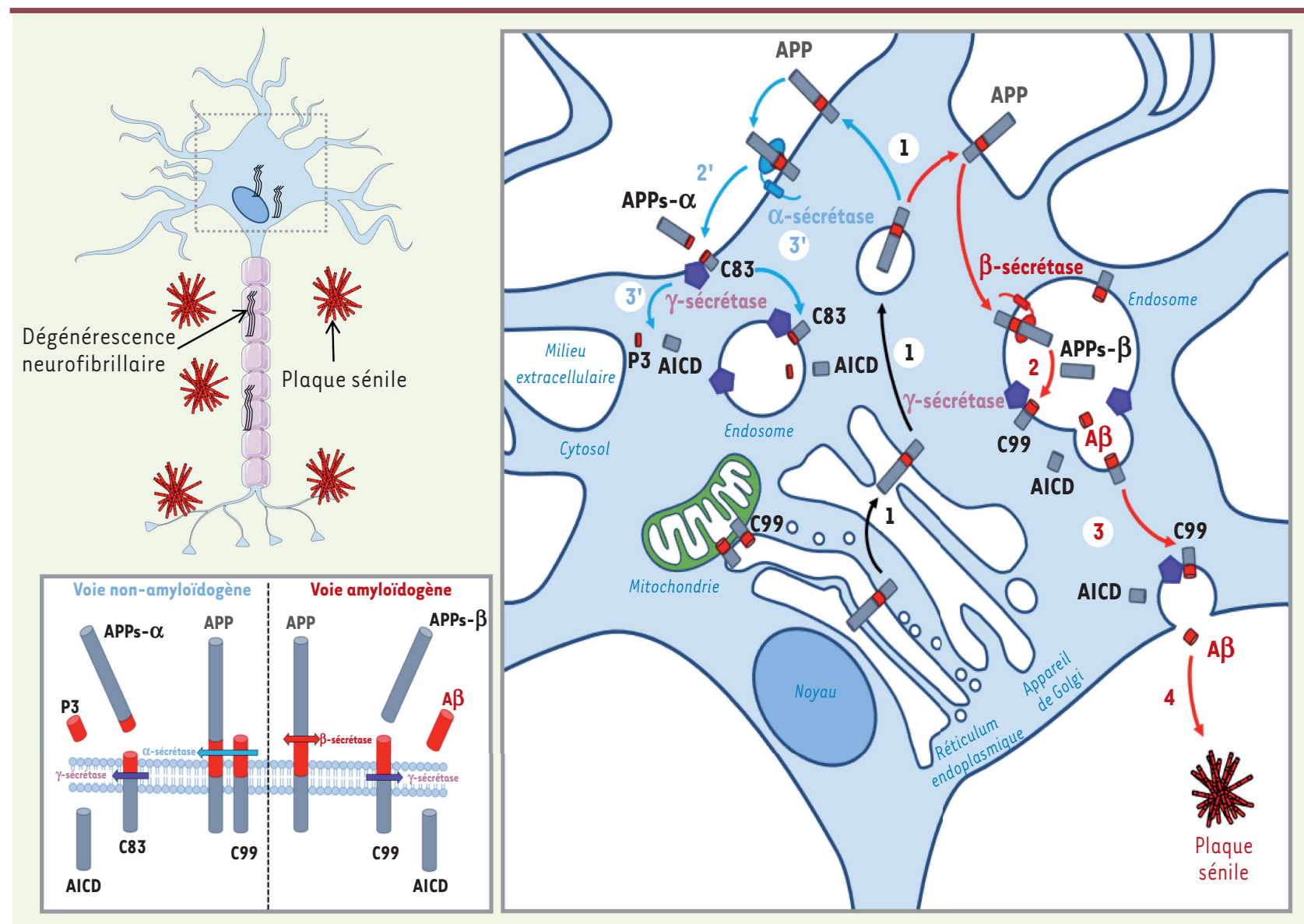

Figure 1. Représentation schématique de deux voies de protéolyse de l'APP : amyloïdogène (1 à 4) et non-amyloidogène ( 1 à $\left.3^{\prime}\right)$. Dans les conditions physiologiques, l'APP mature est adressée à la membrane plasmique (1), produisant, après clivage par l' $\alpha$-sécrétase (2'), le fragment soluble APPs- $\alpha$ et le fragment (83, qui reste ancré à la membrane. Ce dernier est ensuite clivé par la $\gamma$-secrétase (3'), produisant les peptides p3 et AICD (APP intracellular domain). Par ailleurs, l'APP mature (1) est clivée par la $\beta$-sécrétase (2) après son internalisation dans la voie des endosomes/ lysosomes, produisant le fragment soluble APPs- $\beta$, qui est libéré dans l'environnement extracellulaire, et le fragment C99 qui reste ancré dans la membrane et est ensuite clivé par la $\gamma$-secrétase (3), produisant les peptides AICD et A $\beta$. La voie amyloïdogène est favorisée dans les formes familiales de la maladie d'Alzheimer. Dans les formes sporadiques, la baisse de la dégradation des peptides $A \beta$ peut conduire à leur accumulation, à leur agrégation et à la formation des plaques séniles. Nous avons rapporté la présence et le clivage de l'APP dans les membranes associées aux mitochondries, et l'accumulation du fragment 999 dans la mitochondrie [7].

libération des neurotransmetteurs et à la plasticité synaptique. L'homéostasie des mitochondries nécessite leur dégradation par un processus autophagique spécifique, la mitophagie, qui se réalise en trois étapes [3] : 1) la modification (par phosphorylation ou ubiquitination) de certaines protéines mitochondriales pour signaler les mitochondries dysfonctionnelles; 2) la séquestration de la mitochondrie dysfonctionnelle dans une vacuole autophagique (appelée mitophagosome) ; et 3) la fusion de cette vacuole avec les lysosomes contenant des enzymes de dégradation afin de la détruire (Figure 2).

Plusieurs études reposant sur des modèles in vitro et in vivo de la maladie d'Alzheimer [4], et I'analyse post-mortem de cerveaux d'individus atteints de cette maladie à des stades précoces [5], ont montré l'existence d'une altération de la structure du réseau et du métabolisme mitochondrial. Ces défauts ont généralement été associés à la présence du peptide $A \beta$ [4]. L'existence d'un défaut de la mitophagie impliquant principalement
I'A $\beta$ et la protéine Tau hyperphosphorylée a également été soulignée par certains auteurs [6]. Nous avons récemment démontré la présence et le clivage de l'APP dans les «membranes associées aux mitochondries » (mitochondria-associated membranes), des sites de contact entre mitochondries et réticulum endoplasmique, et rapporté la présence d'APP-CTF dans la mitochondrie (Figure 1) [7]. Cette observation nous a conduits à émettre I'hypothèse d'un rôle spécifique de ces APP-CTF dans le dysfonctionne- 


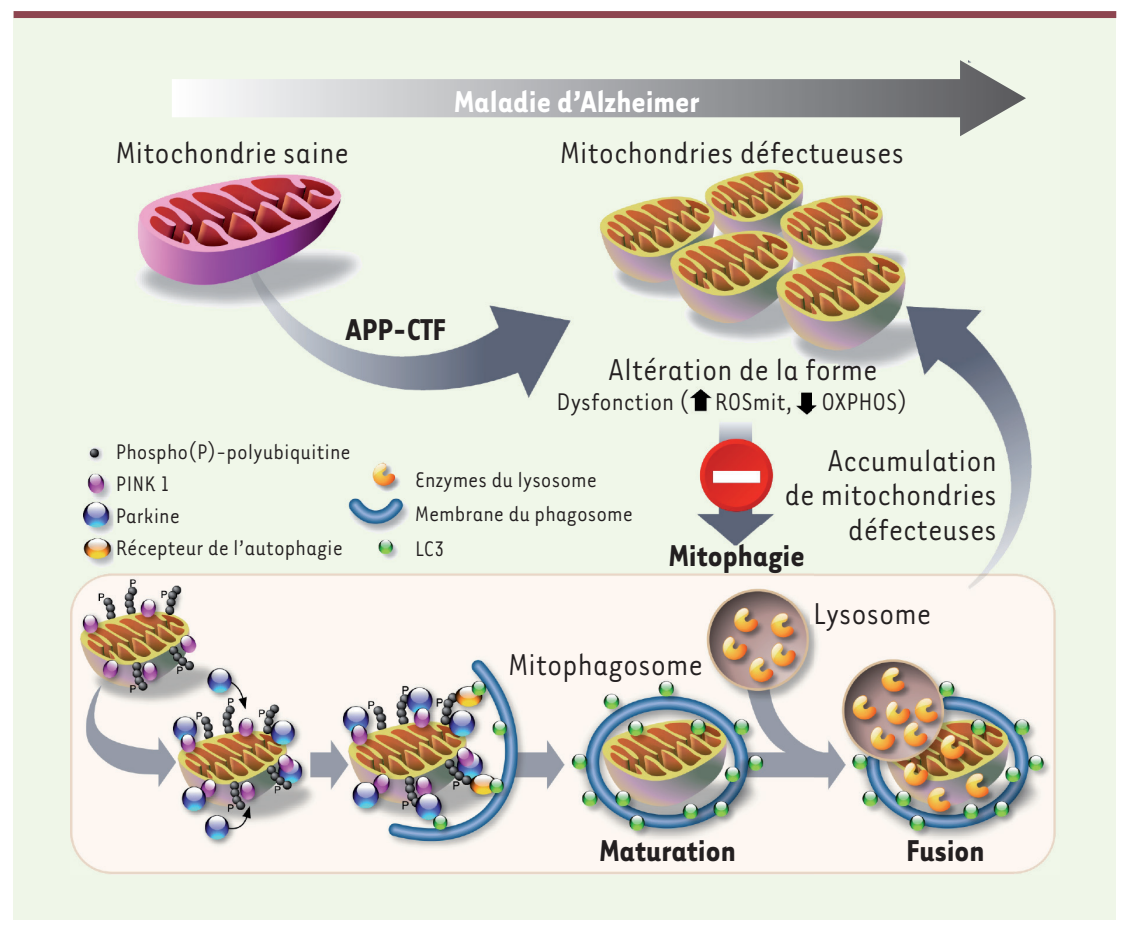

Figure 2. Impact de l'accumulation des fragments APP-CTF sur l'homéostasie mitochondriale dans des modèles d'étude de la maladie d'Alzheimer. L'accumulation des fragments APP-CTF dans la mitochondrie entraîne des altérations spécifiques de sa structure et de sa fonction. Cette accumulation entraîne également une défaillance de la mitophagie, un processus au cours duquel les mitochondries altérées sont habituellement dégradées. La mitophagie dépendante de l'interaction fonctionnelle entre PINKl et la parkine débute par la stabilisation de la protéine PINKI à la surface de la mitochondrie, consécutive à la dépolarisation de la membrane mitochondriale, et son autophosphorylation, entraînant le recrutement de la parkine, une ubiquitine ligase, à la mitochondrie. Cette enzyme assure l'ubiquitination de plusieurs protéines mitochondriales, ce qui favorise le recrutement d'un autophagosome englobant la mitochondrie. Ce mitophagosome mature fusionne avec le lysosome, dont le contenu riche en protéases actives à pH acide permet la dégradation de la mitochondrie et de ses composants. Dans la maladie d'Alzheimer, l'accumulation des fragments APP-CTF induit un blocage de la fusion du mitophagosome avec le lysosome, aboutissant à l'accumulation des mitochondries défectueuses. ROSmit : espèces réactives de l'oxygène dans la mitochondrie; OXPHOS : phosphorylation oxydante.

ment mitochondrial dans la maladie d'Alzheimer [8].

\section{L'accumulation des fragments APP-CTF} induit des défauts de la structure et de la fonction des mitochondries Nous avons utilisé une lignée cellulaire de neuroblastome humain (la lignée SH-SY5Y) dont les cellules surexpriment un APP portant une double mutation décrite dans une famille suédoise, Lys670Met et Asn671Leu (APP Lys670Met, Asn671Leu également appelée $A P P s w e)$, qui favorise l'accumulation des APP-CTF et de I'A $\beta$ [8]. Ces cellules contiennent des mitochondries de plus grande taille, avec une désorganisation de leurs crêtes et une perte de la densité de leur matrice. Afin d'établir la contribution respective de l'A $\beta$ et des fragments APP-CTF dans l'altération de la structure de ces mitochondries, nous avons traité les cellules APPswe avec un inhibiteur de la $\gamma$-sécrétase, bloquant ainsi la production d'A $\beta$ au profit d'une accumulation d'APP-CTF (Figure 1). Par des techniques de fractionnements subcellulaires et d'imagerie confocale, nous avons montré la présence et l'accumulation des APP-CTF dans les mitochondries, associées à une fragmentation du réseau mitochondrial et à une désorganisation et une localisation des crêtes à la périphérie de la matrice mitochondriale. Cette observation a été étayée par le blocage de la $\beta$-sécrétase (réduisant à la fois la production de l'A $\beta$ et du fragment C99). Celui-ci prévient en effet la fragmentation des mitochondries, rétablit la densité de la matrice mitochondriale et normalise l'organisation des crêtes [8]. Parallèlement à l'altération de la structure des mitochondries, nous avons observé que les cellules de la lignée SHSy5y (APPswe) présentent plusieurs signes d'un dysfonctionnement mitochondrial se manifestant par une diminution de la quantité de la sous-unité NDUFB8 (NADH:ubiquinone oxidoreductase subunit B8) du complexe I de la chaîne respiratoire mitochondriale, associée à une baisse de son activité, une diminution du potentiel de membrane mitochondrial $(\Delta \Psi \mathrm{m})$, et une augmentation de la production des espèces réactives de l'oxygène dans la mitochondrie. L'utilisation d'inhibiteurs de la $\beta$-sécrétase et de la $\gamma$-sécrétase nous a permis de discriminer les effets dépendant du peptide $A \beta$ (dépolarisation de la membrane mitochondriale) de ceux dépendant à la fois de l'A $\beta$ et des APPCTF (déficit du complexe I de la chaîne respiratoire mitochondriale). Par ailleurs, nous avons montré que l'accumulation des APP-CTF dans les mitochondries, indépendamment de l'A $\beta$, exacerbe la production des espèces réactives de l'oxygène. Enfin, grâce à l'analyse d'un modèle cellulaire exprimant le fragment C99 de l'APP de façon inductible (cellules SH-SY5Y C99), nous avons confirmé l'impact des APP-CTF sur les dysfonctions mitochondriales. Ce modèle montre en effet une accumulation des APP-CTF dans la mitochondrie, un défaut de l'expression et de l'activité du complexe I de la chaîne respiratoire, et une surproduction des espèces réactives de l'oxygène [8]. 
L'accumulation des fragments APP. CTF induit une défaillance de la mitophagie, indépendamment de l'A $\boldsymbol{\beta}$ L'absence d'un phénotype apoptotique dans les cellules SH-Sy5y APPswe et SHSy5y $C 99$ traitées avec l'inhibiteur de la $\gamma$-sécrétase nous a orientés vers l'étude de la mitophagie dans ces cellules. Par des approches biochimiques, associées à un fractionnement subcellulaire, nous avons quantifié différents marqueurs moléculaires de la mitophagie et avons analysé les différentes étapes du processus mitophagique par l'utilisation de différentes sondes fluorescentes et de l'imagerie. Dans les cellules SH-Sy5y APPswe, nous avons observé les premières étapes du processus mitophagique, caractérisé par un enrichissement en protéine PINKI (PTEN-induced kinase 1) dans la fraction mitochondriale, par le recrutement dans la mitochondrie de la parkine, une ubiquitine ligase, et par la conversion de la forme cytosolique LC3-I de la protéine MAPILC3 (microtubule-associated protein IA/IB-light chain 3) en LC3-II, la forme recrutée à la membrane des autophagosomes. Nos résultats indiquent cependant l'existence d'un blocage du processus mitophagique, révélé par l'absence de dégradation de la protéine SQSTM1/p62 (séquestosome 1 ou protéine de liaison à l'ubiquitine p62) et par l'accumulation de plusieurs protéines mitochondriales (TIMM23, TOMM20, HSP60 et HSP10). Cette défaillance de la mitophagie a été confirmée par la mise en évidence d'un défaut d'adressage et de fusion des mitochondries aux lysosomes. Nous avons ensuite montré que l'accumulation des APP-CTF, indépendamment de l'A $\beta$, exacerbe la défaillance de la mitophagie dans les cellules SH-Sy5y APPswe et SH-Sy5y C99 [8].

L'accumulation des fragments APP-CTF entraîne une altération de la structure des mitochondries et une défaillance de la mitophagie dans des modèles murins de la maladie d'Alzheimer Dans une étude précédente [9], nous avions rapporté une accumulation précoce des fragments APP-CTF (dès l'âge de 3 mois) dans la structure hippocampique du cerveau de souris triple transgéniques ( $3 \times \operatorname{TgAD}$ ) portant la mutation familiale Metl46Val dans le gène codant PSl et surexprimant les protéines humaines APPswe et Tau mutée (Tau Pro301Lys) sous le contrôle du promoteur Thyl.2. Ces souris développent tardivement des plaques séniles (entre 6 et 12 mois) et des NFT (à l'âge de 15 mois). Nos travaux et ceux d'autres équipes ont montré par la suite l'implication des APP-CTF dans le défaut de l'autophagie et dans le déficit synaptique et cognitif $[10,11]$. Nous avons montré l'existence d'une accumulation des APP-CTF dans la fraction mitochondriale chez des souris $3 x \operatorname{Tg} A D$ jeunes (ne présentant pas encore de plaques séniles et n'ayant pas de peptide $A \beta$ détectable), exacerbée par le traitement par l'inhibiteur de la $\gamma$-sécrétase. L'accumulation des APP-CTF induit une altération de la structure des mitochondries se manifestant par une matrice mitochondriale peu dense et par une désorganisation et une localisation des crêtes à la périphérie de la matrice mitochondriale. Ces défauts de la structure des mitochondries sont visibles dès trois mois chez des souris exprimant spécifiquement le fragment (99 par transduction virale (souris AAV-C99). Cette altération de la structure des mitochondries est accompagnée d'une mitophagie défaillante dans les deux modèles murins après le traitement avec l'inhibiteur de la $\gamma$-sécrétase, démontrant le lien entre l'accumulation des APP-CTF in vivo et une accumulation de mitochondries dysfonctionnelles [8].

Nous avons ensuite analysé la structure des mitochondries et la mitophagie dans le modèle $3 x \operatorname{Tg} A D$ à un stade tardif. Une accumulation des APP-CTF, ainsi que les marqueurs histologiques caractéristiques de la maladie d'Alzheimer (plaques séniles et NFT), ont été observés dans les cellules de ce modèle, comparativement aux cellules murines double transgéniques (2xTgAD) qui accumulent les APP-CTF mais ne présentent pas les marqueurs histologiques liés à l'A $\beta$ et pTau [9]. Nous avons montré l'existence, dans les deux modèles, d'une accumulation des APPCTF dans la fraction mitochondriale, associée à une augmentation de la taille des mitochondries et à une organisation anormale de leurs crêtes. Ces résultats démontrent formellement que l'accumulation des APP-CTF est suffisante pour induire des altérations de la structure mitochondriale. Néanmoins, la comparaison de ces deux modèles murins a révélé la contribution tardive de l'A $\beta$ dans l'activation du processus mitophagique chez les souris $3 \times \operatorname{TgAD}$ âgées [8].

\section{L'accumulation des APP-CTF est corrélée avec un défaut de la mitophagie dans les cerveaux de patients atteints de la maladie d'Alzheimer'}

L'analyse biochimique post-mortem des cerveaux de patients atteints de formes non familiales de la maladie d'Alzheimer à des stades avancés démontre une accumulation des APP-CTF (C99 et C83) et de l'A $\beta$ dans la fraction mitochondriale, couplée à un défaut de la mitophagie caractérisé par une baisse des quantités de PINKl et de parkine dans la mitochondrie, une augmentation du ratio LC3-II/LC3-I et de la protéine SQSTM1/p62. Nous avons rapporté une corrélation significative entre les niveaux du fragment C99 et les variations des marqueurs mitophagiques analysés, tandis que ces corrélations sont seulement partielles avec les taux d'A $\beta$ et de pTau. Ce résultat a permis de valider, dans des échantillons humains, les résultats obtenus précédemment dans des modèles d'étude cellulaires et animaux mimant des formes génétiques de la maladie d'Alzheimer.

\section{Perspectives}

Les résultats de notre étude montrent qu'indépendamment de l'action de l'A $\beta$, l'accumulation des fragments APP-CTF 
conduit à une détérioration de la structure et du fonctionnement des mitochondries, et à un blocage du processus de dégradation des mitochondries par mitophagie (Figure 2) [8]. L'accumulation de mitochondries défectueuses associée à la perturbation de la mitophagie est commune à différentes maladies neurodégénératives, telles que la maladie de Parkinson, la maladie de Huntington, la sclérose latérale amyotrophique, et la maladie d'Alzheimer. Le rétablissement d'une fonction mitophagique normale pourrait donc être envisagé comme un nouvel objectif thérapeutique dans la maladie d'Alzheimer et dans d'autres maladies neurodégénératives. $\diamond$ Mitochondrial dysfunction and mitophagy failure in Alzheimer's disease

\section{LIENS D'INTÉRÊT}

Les auteurs déclarent n'avoir aucun lien d'intérêt concernant les données publiées dans cet article.

\section{RÉFÉRENCES}

1. De Strooper B, Karran $\varepsilon$. The cellular phase of Alzheimer's disease. Cell 2016 ; $164: 603-15$.

2. Nhan HS, Chiang K, Koo EH. The multifaceted nature of amyloid precursor protein and its proteolytic fragments: friends and foes. Acta Neuropathol 2015 ; 129 : 1-19.

3. Onishi M, Yamano K, Sato M, et al. Molecular mechanisms and physiological functions of mitophagy. EMBO J 2021 ; 40 : el04705.

4. Atamna H, Frey WH, 2nd. Mechanisms of mitochondrial dysfunction and energy deficiency in Alzheimer's disease. Mitochondrion 2007 ; 7 : 297-310.

5. Manczak M, Park BS, Jung Y, Reddy PH. Differential expression of oxidative phosphorylation genes in patients with Alzheimer's disease: implications for early mitochondrial dysfunction and oxidative damage. Neuromolecular Med 2004 ; 5 : 147-62.

6. Reddy PH, Oliver DM. Amyloid beta and phosphorylated Tau-induced defective autophagy and mitophagy in Alzheimer's disease. Cells $2019 ; 8: 488$.
7. Del Prete D, Suski JM, Oules B, et al. Localization and processing of the amyloid-beta protein precursor in mitochondria-associated membranes. J Alzheimers Dis 2017 ; 55 : 1549-70.

8. Vaillant-Beuchot L, Mary A, Pardossi-Piquard R, et al. Accumulation of amyloid precursor protein C-terminal fragments triggers mitochondrial structure, function, and mitophagy defects in Alzheimer's disease models and human brains. Acta Neuropathol $2021 ; 141$ : 39-65.

9. Lauritzen I, Pardossi-Piquard R, Bauer C, et al. The $\beta$-secretase-derived C-terminal fragment of $\beta A P P, C 99$, but not $A \beta$, is a key contributor to early intraneuronal lesions in triple-transgenic mouse hippocampus. J Neurosci 2012 ; 32 : 16243-55a.

10. Lauritzen I, Pardossi-Piquard R, Bourgeois A, et al. Intraneuronal aggregation of the $\beta$-CTF fragment of APP (C99) induces $A \beta$-independent lysosomalautophagic pathology. Acta Neuropathol 2016; 132 : 257-76.

11. Hung COY, Livesey FJ. Altered gamma-secretase processing of APP disrupts lysosome and autophagosome function in monogenic Alzheimer's disease. Cell Rep 2018 ; 25 : 3647-60 e2.

\section{InI Inserm \\ La science pour la santé From science to health \\ Colloque international Recherche(s), santé et environnement, $\mathrm{XIX}{ }^{\mathrm{e}}-\mathrm{XXI} \mathrm{I}^{\mathrm{e}}$ siècle}

Le Comité pour l'histoire de l'Inserm en partenariat avec Université de Paris, Sorbonne Université et I'UMR SIRICE Avec le soutien de la convention recherche et développement ANSES/CNRS-SIRIC $\varepsilon^{1}$ 16 et 17 novembre 2021 - Amphithéâtre Buffon, UP, Paris $13^{e}$

Sans entrer dans la discussion des faits particuliers, on peut établir comme une loi générale qu'on s'habitue aux intempéries, mais qu'on ne s'accoutume pas aux intoxications. L'homme bien portant peut supporter la chaleur et le froid, affronter le vent et la pluie, l'humidité et les variations de température; mais il n'arrivera jamais à respirer impunément l'air infect, à boire des eaux contaminées, ni à se repaître d'aliments altérés ${ }^{2}$ Jules Rochard, Traité d’hygiène publique et privée, 1897

La crise de la « vache folle », le scandale de l'amiante, ou encore, plus récemment, en septembre 2019, l'incendie de l'usine de produits chimiques Lubrizol de Rouen ont fait et font encore la une des médias ${ }^{3}$. L'environnement est dans le quotidien des individus ; il est tout ce en quoi les individus sont en relation, tout au long de la vie. Global, l'environnement n'est pas pour autant aisément saisissable. Quand il s'agit des questions de santé, l'environnement est bien souvent appréhendé sous les traits du danger et de la catastrophe. Les « particules fines », l'électro-sensibilité, les pesticides ${ }^{4}$, les perturbateurs endocriniens, la question complexe de la relation dose-effet, l'« effet cocktail » constituent la liste non exhaustive des composants médiatiques d'un « monde toxique ». Et si, à l'échelle des populations, il est possible de lier des maladies chroniques à des facteurs environnementaux,-établir avec certitude une causalité à l'échelle individuelle demeure complexe, tant il est difficile de mesurer l'exposition de chacun à des substances dangereuses. Comment protéger la santé d'une menace environnementale diffuse, comment même identifier cette menace ? La santé environnementale contemporaine renvoie à un champ extrêmement large, s'appuyant à la fois sur les sciences du vivant, avec le poids de la toxicologie, et sur les recherches en santé publique à laquelle elle est étroitement liée, et où l'on retrouve le rôle majeur de l'épidémiologie. Alors de quelle(s) recherche(s) essayons-nous de retracer l'histoire? Faisant suite aux journées d'études sur l'épidémiologie et les neurosciences, ce colloque international, organisé par le Comité pour l'histoire de l'Inserm avec la collaboration du Professeur Robert Barouki, en partenariat avec Université de Paris, Sorbonne Université et l'Unité mixte de recherche SIRICE, avec le soutien de la convention recherche et développement ANSES/CNRS-SIRICE, réunira pendant deux jours, des historiens, des chercheurs en sciences sociales, des témoins et des acteurs contemporains. Les échanges qui auront lieu favoriseront, nous l'espérons, le dialogue et une histoire vivante et ouverte de la recherche, de la santé et de l'environnement.

Entrée libre, sur réservation, dans la limite des places disponibles, passe sanitaire obligatoire.

Pour toute information, contactez celine.paillette@ext.inserm.fr

${ }^{1}$ CONVENTION DE RECHERCHE ET DÉVELOPPEMENT_ANSES / CNRS - SIRICE « Santé et sécurité alimentaire au prisme des enjeux scientifiques, environnementaux et commerciaux : Construction politique et sociale d'un système d'expertise et de contrôle dans la France contemporaine ( $x \mid x^{e}-x_{x \mid}{ }^{e}$ siècles) »

2 Jules Rochard, Traité d’hygiène publique et privée, Octave Doin Éditeur, Paris, 1897, préface p. XV.

3 L'incendie a entraîné des mesures de confinement, la cessation de certaines activités agricoles, et la fermeture d'écoles.

4 Expertise collective : «Pesticides et santé - Nouvelles données (2021) 》. Rapport intégral disponible : https://www.inserm.fr/expertise-collective/pesticides-et-sante-nouvelles-donnees-2021/ 